\title{
Ensino de Nutrição Esportiva no Brasil: Proposta de curso de extensão
}

\author{
Sports Nutrition Education in Brazil: Proposal for extension course \\ Enseñanza de Nutrición Deportiva en Brasil: Propuesta de curso de extensión
}

Recebido: 28/03/2021 | Revisado: 03/04/2021 | Aceito: 06/04/2021 | Publicado: 16/04/2021

\author{
Mariana Sudre Lacerda \\ ORCID: https://orcid.org/0000-0002-5526-9678 \\ Centro Universitário de Volta Redonda, Brasil \\ E-mail: marisudre.nutri@hotmail.com \\ Ronaldo Figueiró \\ ORCID: https://orcid.org/0000-0003-0762-1312 \\ Centro Universitário de Volta Redonda, Brasil \\ Centro Universitário Estadual da Zona Oeste, Brasil \\ Universidade Castelo Branco, Brasil \\ E-mail: ronaldofigueiro@gmail.com
}

\begin{abstract}
Resumo
As análises das lacunas envolvendo a disciplina de nutrição esportiva nos 50 melhores cursos de graduação em Nutrição no Brasil, informação que foi divulgada através de ranking pelo INEP em 2016, culminaram em uma proposta de curso de extensão. Podemos observar que nas diretrizes curriculares nacionais do curso de graduação em nutrição, instituídas pelo Conselho Nacional de Educação, a disciplina de nutrição esportiva não é considerada como obrigatória. Tal fato nos permite supor que, embora o número de nutricionistas seja expressivo no Brasil, há pouca atuação dos profissionais na área esportiva, como um reflexo de uma deficiência do ensino nessa área. Diante deste cenário, o curso de extensão criado seria um facilitador no processo de formação dos discentes, permitindo a abordagem de temas relacionados a nutrição esportiva. O curso ainda visa discutir atuais mitos envolvendo suplementação, preparar os profissionais para atendimento de atletas de alta performance, indivíduos praticantes de exercícios físicos e o desenvolvimento de censo crítico dos discentes durante a avaliação nutricional.
\end{abstract}

Palavras-chave: Educação; Nutrição esportiva; Curso de extensão; Ensino.

\begin{abstract}
The analysis of the gaps involving the discipline of sports nutrition in the 50 best undergraduate courses in Nutrition in Brazil, information disclosed through a ranking by INEP in 2016, culminated in a proposal for an extension course. We can observe that in the national curricular guidelines of the undergraduate course in nutrition, instituted by the National Education Council, the discipline of sports nutrition is not considered as mandatory. This fact allows us to suppose that, although the number of nutritionists is significant in Brazil, there is little performance by professionals in the sports field, as a reflection of a teaching deficiency in this area. In view of this scenario, the extension course created would be a facilitator in the process of training students, allowing the approach of topics related to sports nutrition. The course also aims to discuss current myths involving supplementation, prepare professionals to care for high-performance athletes, individuals who practice physical exercises and the development of students' critical sense during nutritional assessment.
\end{abstract}

Keywords: Education; Sports nutrition; Extension course; Teaching.

\section{Resumen}

El análisis de las brechas que involucran la disciplina de nutrición deportiva en los 50 mejores cursos de pregrado en Nutrición en Brasil, información divulgada a través de un ranking del INEP en 2016, culminó en una propuesta de curso de extensión. Podemos observar que en los lineamientos curriculares nacionales de la carrera de pregrado en nutrición, instituidos por el Consejo Nacional de Educación, la disciplina de nutrición deportiva no se considera obligatoria. Este hecho permite suponer que, si bien el número de nutricionistas es significativo en Brasil, hay poco desempeño de los profesionales en el campo deportivo, como reflejo de una deficiencia docente en esta área. Ante este escenario, el curso de extensión creado sería un facilitador en el proceso de formación de los estudiantes, permitiendo el abordaje de temas relacionados con la nutrición deportiva. El curso también tiene como objetivo discutir los mitos actuales relacionados con la suplementación, preparar a los profesionales para cuidar de los deportistas de alto rendimiento, las personas que practican ejercicios físicos y el desarrollo del sentido crítico de los estudiantes durante la evaluación nutricional.

Palabras clave: Educación; Nutrición deportiva; Curso de extensión; Enseñanza. 


\section{Introdução}

A evolução da ciência em diversas áreas do conhecimento humano e também da sociedade tem proporcionado um novo modelo de coletividade. Com isso, tem-se observado a formação universitária não só como condição para inserção no mercado de trabalho, como também um direito do indivíduo a cidadania. A sociedade tem questionado o andamento do ensino superior no que tange as finalidades de seus programas, tais como: construir a habilidade para resolver problemas, incentivar a análise crítica e estabelecer uma abordagem criativa (Costa, 2009).

O fenômeno da ascendência da Nutrição como ciência, política social e profissão, no cenário mundial, é relativamente recente e iniciado no século XX. O princípio ocorreu no período entre guerras, através da observação de alguns países quanto ao rendimento mais eficaz dos soldados bem alimentados. A partir disso, hipóteses e experimentos foram iniciados. Pedro Escudero, em 1926, foi pioneiro na criação do Instituto Nacional de Nutrição. Apenas em 1939 o primeiro curso foi inaugurado no Brasil (Vasconcelos, 2002).

Diante do importante desenvolvimento industrial ocorrido na década de 60, que também englobou a área de saúde, a profissão de nutricionista foi regulamentada em 24 de Abril de 1967. No dia 8 de Junho de 1980 houve a criação da Associação Brasileira de Nutrição (ASBRAN) e, decorrente dela, o surgimento de novas áreas de atuação do nutricionista, tais como: esportiva, marketing, hotelaria e atendimento domiciliar (Cristofolli et al. 2017).

A área de nutrição esportiva tem sido citada por vários trabalhos que buscam analisar não só o perfil dos consumidores de suplementos alimentares em ambientes esportivos, como também quem os indicam e quais os tipos. Encontramos concordância nos trabalhos de Borges et al. (2016), Karkle (2015) e Bertoletti et al. (2016), pois mais da metade dos entrevistados consumiam suplementos alimentares sem a prescrição de um nutricionista. Constatou-se que os suplementos alimentares mais consumidos foram os proteicos nos trabalhos de Rodrigues e Chaves (2016), Santos e Pereira (2017), Cipriano e Fabris (2016) e Ferreira e Quintão (2016). De acordo com o estudo de Nunes e Gonçalves (2017) contemplando 169 estudantes de Educação Física, a fim de averiguar qual a fonte mais utilizada para se obter informações sobre recursos ergogênicos nutricionais (incluindo suplementação), a internet foi a mais citada.

Em estudo realizado por Benetti e Chagas (2017), foi relatado que $45 \%$ da fonte de indicação de suplementos alimentares procedeu de educadores físicos, o que também foi observado na pesquisa de Belini et al. (2015), pois a indicação por educadores físicos foi maior do que a de nutricionistas. Deste modo, fica perceptível a necessidade de nutricionistas que atuem na área de nutrição esportiva.

A última pesquisa realizada pelo CFN, no segundo trimestre de 2018, verificou que existem 131.737 nutricionistas no Brasil, dados estes que nos possibilitam avaliar o Ensino nessa área. Além disso, em outra pesquisa realizada pelo CFN (2016), tendo a participação de 1.104 nutricionistas empregados, podemos observar as áreas de atuação deles. Os resultados foram os seguintes: 30,8\% atuam em alimentação coletiva, 30,4\% em nutrição clínica, 17,7\% em saúde coletiva, 11,4\% em docência, 2,6\% em indústria, 2,5\% em nutrição esportiva, 1,3\% em marketing e 3,3\% outros. A partir dos dados levantados pelo CFN, podemos questionar: Por que, apesar do número expressivo de nutricionistas no Brasil, ainda temos pouca atuação destes na área de Nutrição Esportiva? Quais as dificuldades encontradas na prática docente?

O objetivo deste estudo é propor a elaboração de um curso de extensão de modo a contribuir para a formação discente no processo ensino aprendizagem, através de assuntos abordados na disciplina de nutrição esportiva.

\section{Metodologia}

O presente estudo configura-se como uma pesquisa de natureza quantitativa e qualitativa, que utiliza o levantamento inicial de dados para interpretações e análises futuras. Os métodos quantitativos e qualitativos auxiliam no entendimento de aspectos essenciais e lógicos de um determinado fenômeno ou fato estudado. Entender sobre a forma de utilização desses 
métodos possibilita uma análise correta, eficaz e produtiva de dados, pois o conhecimento é construído de forma pensada e planejada (Proetti, 2017). Para se chegar à proposta de elaboração do curso de extensão, foram analisadas as matrizes curriculares de 50 cursos de graduação em Nutrição no Brasil. Esta informação foi retirada do site do Instituto Nacional de Estudos e Pesquisas Educacionais Anísio Teixeira (INEP, 2016). A partir dos dados encontrados no site, foi feita uma seleção dos 50 melhores cursos de graduação em Nutrição no Brasil, resultando em um ranking com base nas maiores notas encontradas para conceito preliminar de curso (CPC).

A pesquisa documental foi realizada através dos sites correspondentes de cada curso para entender a distribuição geográfica deles e também a prevalência da disciplina de nutrição esportiva nas grades curriculares. A partir desta análise e de uma revisão bibliográfica sobre o ensino de nutrição esportiva, se constrói uma discussão acerca do cenário atual dos cursos. Traçado o panorama dos cursos, foram analisadas lacunas envolvendo a disciplina de nutrição esportiva e, a partir deste panorama, uma proposta de curso de extensão foi idealizada.

\section{Resultados e Discussão}

\subsection{Nutrição esportiva como componente curricular}

O CPC é um indicador de qualidade dos cursos superiores que é mais completo que o conceito Enade, pois leva em consideração o corpo docente, infraestrutura, recursos didáticos pedagógicos e demais insumos no momento da avaliação. Este indicador contempla cursos presenciais e a distância, juntando os resultados dos alunos das duas modalidades (INEP, 2019).

No ano de 2016 foram avaliados 4.300 cursos pelo INEP e dentre eles, 333 foram de Nutrição. Apenas 7 cursos de Nutrição obtiveram a nota máxima. Levando em consideração o ranking que contempla os 50 melhores cursos de Nutrição avaliados, podemos verificar que 74\% apresentam a disciplina de nutrição esportiva como componente curricular (Figura 1):

Figura 1 - Percentual de cursos que apresentam a disciplina de Nutrição esportiva.

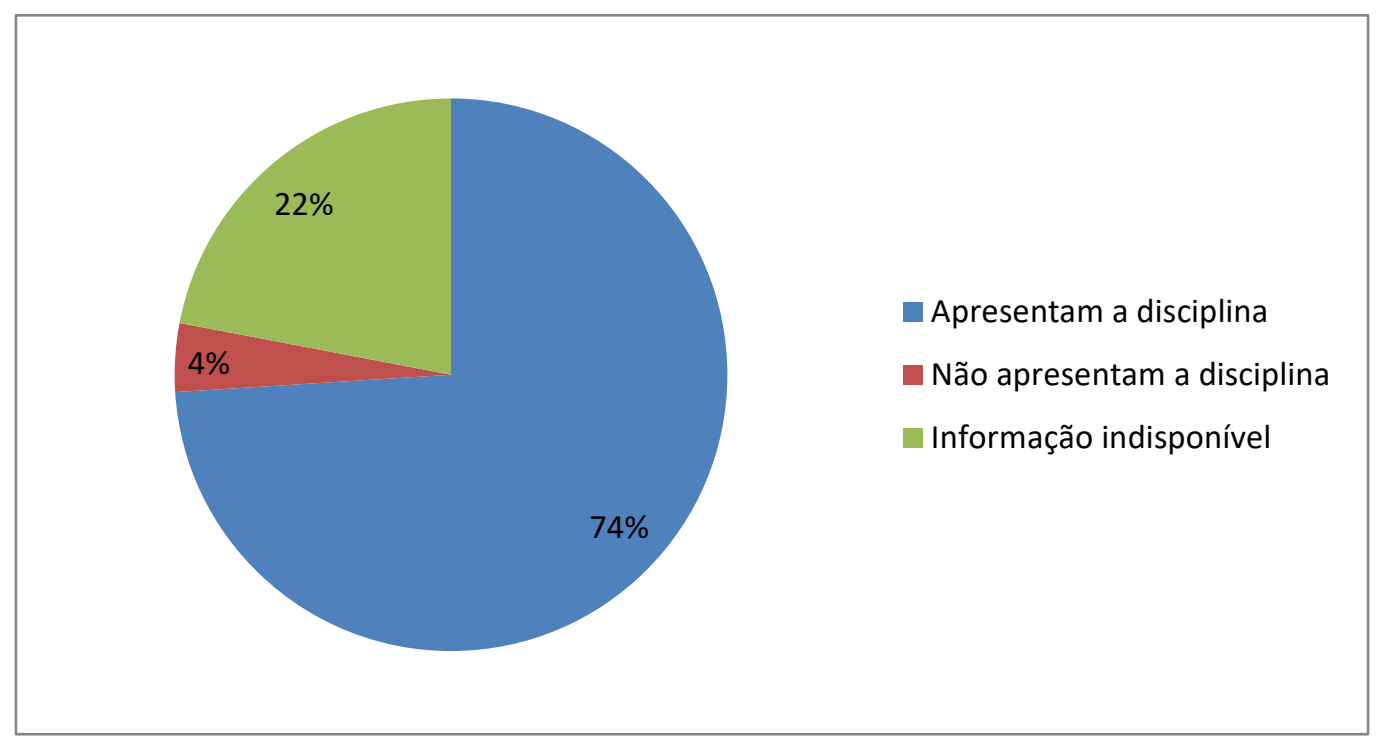

Fonte: Autores.

Observou-se que dentre os cursos investigados, um não apresentou a disciplina de nutrição esportiva na grade curricular, ainda que tenha obtido nota máxima no CPC. Os demais cursos que obtiveram nota máxima na avaliação apresentaram a disciplina envolvendo nutrição esportiva com nomenclaturas diferentes. As nomenclaturas encontradas foram: nutrição esportiva, nutrição no esporte, nutrição aplicada ao exercício e ao esporte e nutrição aplicada a atividade física. Vale destacar que, apesar da nota máxima obtida, apenas uma faculdade apresentou estágio obrigatório nessa disciplina. 
No ranking dos 50 melhores cursos de nutrição avaliados, $74 \%$ destes apresentaram a disciplina de nutrição esportiva como eletiva, $4 \%$ não apresentaram a disciplina na grade do curso e $22 \%$ não apresentavam a informação disponível para consulta pública no sítio oficial da instituição.

Dentre os cursos que não apresentaram a disciplina como eletiva, apenas um apresentou como optativa e outro apresentou estágio em nutrição esportiva como componente curricular. É importante salientar que o ranking apresenta cursos de todo o Brasil e até mesmo os mais bem conceituados não possuem a disciplina na grade. Desta forma, fica evidenciado que existem cursos que possuem a disciplina de nutrição esportiva obrigatória e que não alcançaram a nota máxima no IGC.

Dentre os cursos que figuram nas 50 primeiras posições do ranking, 36 (72\%) pertenciam à região Sudeste, enquanto 9 (18\%) estavam situados na região Sul, 4 (8\%) na região Nordeste e apenas um na região Centro-Oeste (2\%) (Figura 2), sendo assim possível observar que a maioria dos cursos estão concentrados da região Sudeste.

Figura 2 - Distribuição geográfica dos artigos analisados.

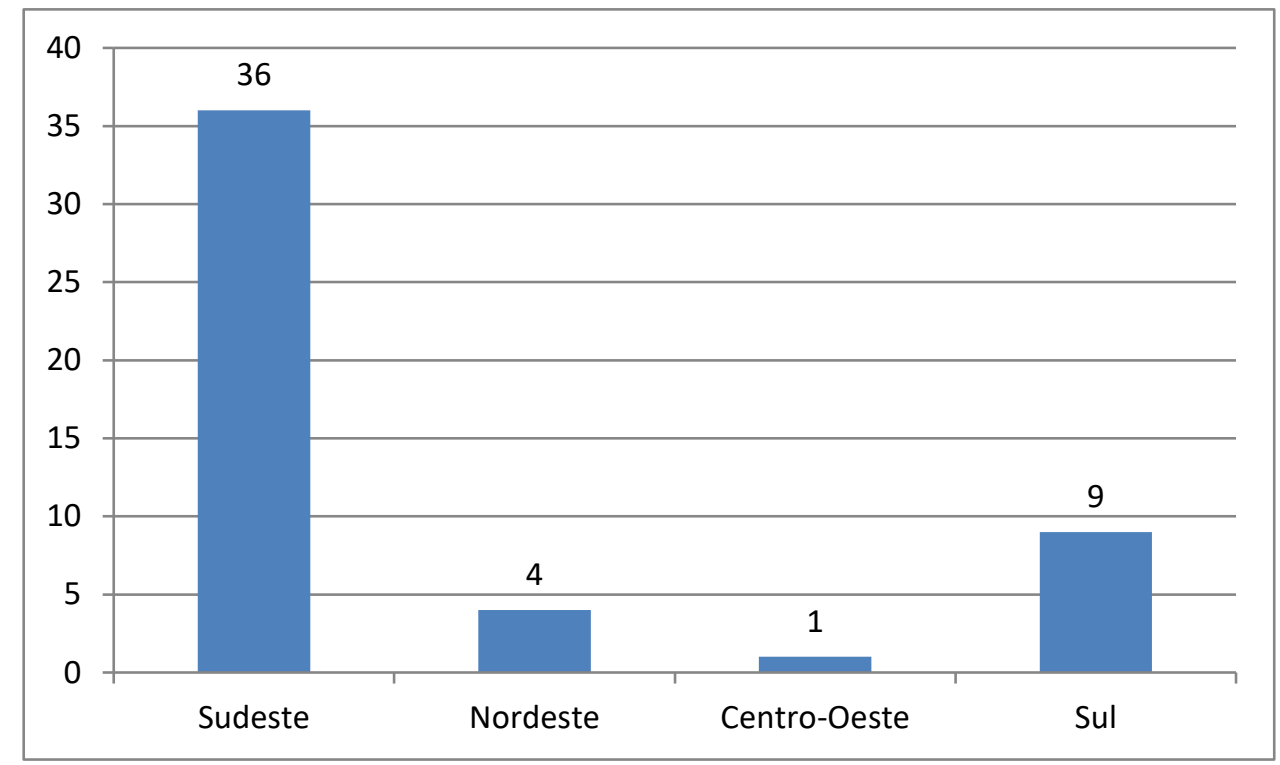

Fonte: Autores.

Com relação a carga horária da disciplina nas instituições em que ela existe, não há uma uniformidade. A variação ficou entre 30, 40 e 60 horas, sendo 37,5\% dos cursos apresentando 60 horas na grade curricular, 18,75\% apresentando 40 horas e 12,5\% apresentando 60 horas, tornando possível observar que, ainda que os cursos tenham a disciplina na grade, a prevalência de cursos com a menor carga horária foi maior. Interessante salientar que, $31,25 \%$ dos cursos não apontaram a carga horária da disciplina nos sites.

Vale ressaltar que, segundo as Diretrizes Curriculares Nacionais para o Curso de Graduação em Nutrição (2001), tanto a disciplina de Nutrição esportiva como o estágio não são obrigatórios, sendo facultativo às instituições aderir. Portanto, cada curso de graduação em Nutrição deverá ter um projeto pedagógico, buscando a formação integral do estudante através de uma adequação entre o ensino, a pesquisa e a extensão/assistência. Desta forma, em decorrência da falta de valorização da área na grade curricular do curso de nutrição, os praticantes de exercício físico ficam vulneráveis a orientações inadequadas de indivíduos desqualificados.

Podemos verificar no estudo de Lima et al. (2019) que em relação aos cursos de Nutrição do Centro-oeste avaliados, $100 \%$ deles possuem a disciplina de nutrição esportiva como obrigatória. Em contrapartida, a carga horária da disciplina teve 
muita variação de um curso para outro. Resultados diferentes foram encontrados na pesquisa de Silveira et al. (2019), onde apenas $88,6 \%$ dos entrevistados relataram existir a disciplina de nutrição esportiva no curso de nutrição.

Entretanto, é controverso o fato de que, segundo a Associação Brasileira de Empresas de Produtos Nutricionais (ABENUTRI), o mercado de suplementos alimentares movimentou só em 2018, R \$ 2,2 bilhões no Brasil. Este valor é equivalente a $12 \%$ de aumento comparado com o ano anterior, e a expectativa para 2019 é que o crescimento seja em torno de $15 \%$. Deste modo, fica explícito que a indústria tem investido e lucrado com estes produtos, e, ao mesmo tempo, ainda não temos profissionais qualificados no mercado que sejam capazes de suprir esta demanda.

\section{2 $O$ ensino de nutrição}

Um estudo realizado por Luz et al. (2015) avaliou a formação do profissional de nutrição na percepção dos docentes nutricionistas de uma instituição de Ensino superior do nordeste brasileiro. A maioria dos participantes referiu regime de trabalho em dedicação exclusiva e quanto à titulação, 70\% dos docentes informaram possuir mestrado (25\%) ou doutorado (45\%).

Dando continuidade, no trabalho de Moraes (2014), foi evidenciado que há uma visão por parte do docente de que o domínio de conhecimentos específicos é suficiente para o exercício da docência, nesse nível de ensino. Essa visão reflete uma noção de que a docência universitária não requer como nos demais níveis de ensino, formação específica ou continuada que se resulte em espaços para reflexões e trocas a respeito do exercício da docência.

No trabalho realizado por Costa (2016), foi observado que as maiores dificuldades encontradas na prática docente consistiam em: falta de material, dificuldade dos alunos em absorver o conteúdo e falta de tempo para preparar as aulas.

Podemos verificar dificuldades semelhantes no trabalho de Cardoso et al. (2016), no qual o enfrentamento inicial dos docentes foi: a insegurança, relacionamento com alunos e colegas, falta de infraestrutura e de formação pedagógica. Por outro lado, facilidades com a docência foram: domínio do conteúdo, aptidão para falar em público, acolhimento dos colegas e gostar da docência. A maioria dos docentes não teve formação pedagógica, indo ao encontro do trabalho de Moraes (2014), onde, além disso, os motivos que levaram à escolha da profissão foram a influência familiar e de antigos mestres, a vocação e a necessidade financeira.

\subsection{Proposta de curso de extensão}

Como podemos verificar, existem lacunas no ensino de Nutrição Esportiva que podem ser preenchidas através de propostas que auxiliem no processo ensino aprendizagem. Desta forma, sugerimos a elaboração de um curso de extensão. Este teria uma carga horária total de $14 \mathrm{~h}$, sendo ministrado Sábado e Domingo. Teria início às 08h no Sábado, com pausa 12h para almoço e retornando às $13 \mathrm{~h} 30$, com término às $17 \mathrm{~h} 30$. Já no Domingo, teria início às $09 \mathrm{~h}$, com pausa $12 \mathrm{~h}$ para almoço e retornando às $13 \mathrm{~h} 30$, com término às $16 \mathrm{~h} 30$.

O curso visa a formação de nutricionistas capazes de se inserir no mercado de trabalho para executar atendimentos não só a atletas profissionais e amadores, como também de praticantes de atividade física em consultórios, academias e clubes. Após a conclusão do curso, o profissional estaria apto a compreender e aprofundar os conhecimentos relacionados à nutrição e aos aspectos intrínsecos do funcionamento do organismo humano com ênfase nas características e alterações fisiológicas e nutricionais decorrentes da prática esportiva. Teríamos como público alvo nutricionistas e estudantes de Nutrição.

Alguns tópicos foram pensados como ementa, conforme Quadro 1 a seguir: 
Quadro 1 - Ementa do curso de extensão em nutrição esportiva.

\begin{tabular}{|l|l|}
\hline \multicolumn{2}{|c|}{ EMENTA } \\
\hline 1 - Introdução a Nutrição Esportiva & 6 - Hidratação \\
\hline 2 - Demanda energética no exercício físico & 7 - Recursos ergogênicos nutricionais \\
\hline $\begin{array}{l}3 \text { - Planejamento dietético para praticantes de } \\
\text { exercício físico }\end{array}$ & 8 - Mitos da suplementação \\
\hline $4-$ Avaliação da composição corporal de atletas & 9 - Legislações \\
\hline 5 - Refeição pré, durante e pós treino & 10 - Resoluções \\
\hline
\end{tabular}

Fonte: Autores.

Para os objetivos, foram propostos: identificar a relação da nutrição com o desempenho esportivo, reconhecer os papéis de cada micro e macronutriente na prática esportiva, expor a classificação dos suplementos alimentares e suas aplicações na prática, estimar o gasto energético total de pessoas ativas, discutir sobre os mitos a respeito da suplementação nutricional, identificar a importância da hidratação em esportes específicos e conhecer a RDC $\mathrm{N}^{\circ}$ 18, DE 27 DE ABRIL DE 2010 - Regulamento Técnico sobre Alimentos para Atletas e Resolução CFN N 600, de 25 de Fevereiro de 2018. Os recursos didáticos seriam: data show, artigos científicos e livros. Segue abaixo modelo de cronograma (Quadro 2) para as atividades:

Quadro 2 - Cronograma das atividades.

\begin{tabular}{|l|l|}
\hline \multicolumn{1}{|c|}{ SÁBADO } & \multicolumn{1}{|c|}{ DOMINGO } \\
\hline 08h00: Introdução a Nutrição & 09h00: Hidratação \\
\hline 09h00: Metabolismo energético e exercício físico & 10h00: Recursos ergogênicos nutricionais \\
\hline Coffee break: 15 minutos & Almoço: 12h às 13h30 \\
\hline $\begin{array}{l}\text { 10h15: Planejamento dietético para praticantes de } \\
\text { exercício físico }\end{array}$ & 13h30: Legislações e resoluções \\
\hline Almoço: 12h às 13h30 & 14h30: Mitos da suplementação \\
\hline 13h30: Avaliação física e antropométrica & 16h30: Encerramento \\
\hline 15h00: Refeição pré, durante e pós treino & \\
\hline 17h00: Dúvidas e perguntas & \\
\hline 17h30: Encerramento & \\
\hline
\end{tabular}

Fonte: Autores.

\section{Conclusão}

Podemos observar a deficiência de normas e diretrizes que considerem a disciplina de nutrição esportiva como obrigatória. Tal fato nos permite supor que embora o número de nutricionistas seja expressivo no Brasil, há pouca atuação dos profissionais na área esportiva como um reflexo de uma deficiência do ensino nessa área. Diante deste cenário, o curso de extensão seria um facilitador no processo de formação dos discentes, permitindo a abordagem de temas relacionados a nutrição esportiva.

Esperamos que o presente estudo contribua para trabalhos futuros, em que sejam abordadas outras variáveis, a fim de possibilitar a identificação de novas ferramentas que reforcem e incentivem a temática sobre nutrição esportiva no período da graduação. 


\section{Referências}

Belini, M. R. L., Silva, M., \& Gehring, L. (2015). Utilização de suplementos alimentares por adolescentes praticantes de musculação em academias da cidade de Campo Mourão - PR. Revista Brasileira de Nutrição Esportiva, 9 (54), 553-562.

Benetti, F., \& Chagas, B. C. (2017). Avaliação do consumo de suplementos alimentares por praticantes de musculação das academias do Município de Tenente Portela - RS. Revista Brasileira de Nutrição Esportiva, 11 (63), 363-374.

Bertoletti, A. C., Santos, A., \& Benetti, F. (2016). Consumo de suplementos alimentares por praticantes de musculação e sua relação com o acompanhamento nutricional individualizado. Revista Brasileira de Nutrição Esportiva, 10 (58), 371-380.

Borges, N. R., Silva, V. S. F., \& Rodrigues, V. D. (2016). Consumo de suplementos alimentares em academias de Montes Claros / MG. Revista Multitexto, 4 (01), 54-59.

Cardoso, C. G. L. V., Costa, N. M. S. C., \& Moraes, B. A. (2016). Desafios da formação pedagógica em nutrição. Revista ciência, docência y tecnología, Argentina, 27 (53).

Cipriano, D. B., \& Fabris, F. M. (2016). Avaliação nutricional de praticantes de musculação de uma academia do centro de Criciúma - SC. Revista Brasileira de Nutrição Esportiva, 10 (58), 402-407.

Costa, N. M. S. C. (2009). Formação pedagógica de professores de nutrição: uma omissão consentida? Revista de Nutrição, 22 (1).

Costa, C. M. (2016). Percepção dos professores do curso de Nutrição sobre suas dificuldades na prática docente. Revista Brasileira De Assistência Interdisciplinar, 2 (2)

Cristofolli, C., Bonato, L., \& Ravazzani, E. D. A. (2017). Análise histórica da profissão de nutricionista. Cadernos da Escola de Saúde, 2 (6), 1-7.

Conselho Federal de Nutricionistas (2018). http://pesquisa.cfn.org.br//.

Conselho Federal de Nutricionistas (2016). https://pesquisa.cfn.org.br//.

CNE/CES N $\mathrm{N}^{\mathrm{o}}$, De 7 De Novembro de 2001. Diretrizes curriculares nacionais do curso de graduação em Nutrição. http://portal.mec.gov.br/cne/arquivos/pdf/CES05.pdf/.

Ferreira, C. S., \& Quintão, D. F. (2016). Prevalência do uso de suplementos alimentares por praticantes de exercício físico em uma academia da cidade de Cataguases-MG. Revista Brasileira de Nutrição Esportiva, São Paulo, 10 (59), 507-517.

INEP (2019). http://portal.inep.gov.br/conceito-preliminar-de-curso-cpc-

Karkle, M. B. (2015). Uso de suplemento alimentar por praticantes de musculação e sua visão sobre o profissional nutricionista na área de nutrição esportiva em uma academia no município de Braço do Norte - SC. Revista Brasileira de Nutrição Esportiva, São Paulo, 9 (53), $447-453$.

Lima, A. M. S., Rocha, T. E. S., \& Costa, T. N. F. (2019). Nutrição Esportiva como Componente Curricular nos cursos de Nutrição e Educação Física do Centrooeste do Brasil. Revista Brasileira de Ciências da Saúde, 23 (1).

Luz, M. M. A. et al (2015). A formação do profissional nutricionista na percepção do docente. Revista Interface, 19 (54).

Moraes, P. J. B. (2014). De nutricionista a professor: o percurso formativo dos atuais docentes do curso de Nutrição. Dissertação (Mestrado em Educação) Universidade Cidade de São Paulo, São Paulo.

Nunes, L. H., \& Gonçalves, A. (2017). Consumo e nível de conhecimento sobre recursos ergogênicos entre estudantes de Educação Física. Revista Brasileira de Nutrição Esportiva, São Paulo, 11 (67), Suplementar 1, 875-883.

Proetti, S. (2017). As pesquisas qualitativa e quantitativa como métodos de investigação científica: um estudo comparativo e objetivo. Revista Lumen, 2 (4), 123.

Resolução CNE/CES No 5 (2001). Diretrizes Curriculares Nacionais para o Curso de Graduação em Nutrição. Ministério da Educação.

Rodrigues, A. L. P., \& Chaves, R. F. (2017). Consumo de suplementos alimentares por praticantes de musculação em uma academia de Fortaleza - CE. Revista Brasileira de Nutrição Esportiva, 10 (60), 596-602.

Santos, E. A., \& Pereira, F. B. (2017). Conhecimento sobre suplementos alimentares entre praticantes de exercício físico. Revista Brasileira de Nutrição Esportiva, 11 (62), 134-140.

Silveira et al. (2019). Conhecimento de acadêmicos de Nutrição sobre alimentação saudável e nutrição esportiva. Revista Brasileira de Nutrição Esportiva, 13 (78), 227-235.

Simpósio Suplementos na Mídia. Blog ABENUTRI. (2019). http://www.abenutri.org/blog/

Vasconcelos, F. A. G. (2002). O nutricionista no Brasil: uma análise histórica. Revista de Nutrição, 15 (02). 